\title{
Concerns in pregnancy and childbirth of women with inflammatory bowel disease
}

\author{
Sung-Ae Jung \\ Department of Internal Medicine, Ewha Womans University School of Medicine, Ewha Medical Research Institute, Seoul, Korea
}

\section{Article: Pregnancy outcome in women with inflammatory bowel disease treated with anti-tumor necrosis factor and/or thiopurine therapy: a multicenter study from Japan (Intest Res 2016;14:139-145)}

Inflammatory bowel disease (IBD) is a group of conditions that cause chronic inflammation of the digestive tract. Many women with IBD are in the peak of their reproductive years. They are likely to go through pregnancy and childbirth during their illness. In particular, poorly controlled IBD is well known to be associated with a number of adverse outcomes such as spontaneous abortion, low birth weight, small for gestational age, preterm delivery, and congenital anomaly. To achieve good outcomes, women with IBD who are planning pregnancy or are pregnant must be treated and followed up by using a multidisciplinary approach that involves gastroenterologists and obstetricians who specialize in high-risk mothers.

Gastroenterologists must be responsible for the selection of safe and effective medications for both the fetus and mother. The definite data available on IBD medications that are safe to use during pregnancy are scarce. Most sources of evidence were from Western countries, not Asian countries. In addition, some data were obtained based on evidence in women with rheumatoid disease, who use similar medications as patients with IBD.

Currently, most IBD medications are considered low-risk and can now be used during pregnancy and breastfeeding,

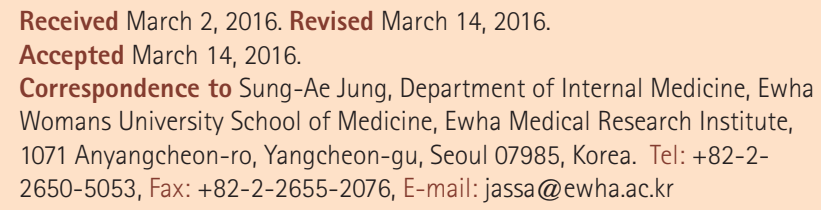

Financial support: None. Conflict of interest: None. except for methotrexate. Among the anti-tumor necrosis factor alpha agents (anti-TNF $\alpha$ ), infliximab, adalimumab, and certolizumab pregol are approved for CD, and infliximab, adalimumab, and golimumab are approved for UC. ${ }^{1}$ However, anti-TNF $\alpha$ agents such as infliximab and adalimumab may be administered as early as the beginning of the second trimester. $^{2}$ As a result of fetal drug exposure and immune development and infectious risk, the optimal dosing and discontinuance timing of anti-TNF medication during pregnancy have been controversial. The dosing and discontinuation timing of anti-TNF medication vary from individual to individual and are still under investigation. ${ }^{3}$ Even though no definite evidence of birth defects or other adverse outcomes has been found, an increased risk of infection and immunity defects have been demonstrated by anti-TNF $\alpha{ }^{4}$

In studies about pregnancy outcomes of the administration of thiopurine immunomodulators, 6-mercaptopurine, and the prodrug azathioprine, no birth defects were reported. ${ }^{5,6}$ However, we must consider that these medications are under Food and Drug Administration category D, and our experiences are limited.

The recent study by Komoto et al. was interesting in that it provided the first data from Asians about the examination of the outcomes of pregnancy in patients with IBD treated with anti-TNF and/or thiopurine. ${ }^{7}$ However, this study left several points that need to be addressed. As the authors already mentioned, it was difficult to design and ensure an even distribution of study population according to the type of medication, subtype of IBD, and disease activity. In addition, the

\footnotetext{
๑ Copyright 2016. Korean Association for the Study of Intestinal Diseases. All rights reserved.

This is an Open Access article distributed under the terms of the Creative Commons Attribution Non-Commercial License (http://creativecommons.org/licenses/by-nc/4.0)

which permits unrestricted non-commercial use, distribution, and reproduction in any medium, provided the original work is properly cited.
} 
authors compared the rates of adverse outcomes with those in the normal population. They concluded that the incidence rates of low birth weight and preterm birth in women with IBD were higher than those in the normal population, regardless of the use of anti-TNF or thiopurine. In addition, they reported that the incidence of congenital malformation was comparable with that in the general population, regardless of the use of anti-TNF or thiopurine. However, the following assumptions are necessary for analysis: First, the small number of patients $(\mathrm{n}=72)$ in this study must be representative of the entire IBD population. Second, its results must be within the acceptable CI. Therefore, these conclusions must be supported by additional evidence in the future. In addition, considering the association between fetal drug exposure and concern for potential risks, the period of fetal drug exposure should be considered in the analysis. Third, to assess the causality of adverse outcomes, analysis between two groups, such as between the group with and that without $\mathrm{d}$ adverse outcomes, is necessary.

It remains difficult to conclude whether anti-TNF $\alpha$ or thiopurine caused adverse outcomes. Nevertheless, data from this study were valuable, as these were the first data obtained from Asian populations. In addition, this study may draw attention toward patients with IBD during pregnancy. Furthermore, large-scale, nationwide studies are necessary to clarify the safety of IBD medication.

\section{REFERENCES}

1. Mahadevan U, Matro R. Care of the pregnant patient with inflammatory bowel disease. Obstet Gynecol 2015;126:401-412.

2. Gisbert JP, Chaparro M. Safety of anti-TNF agents during pregnancy and breastfeeding in women with inflammatory bowel disease. Am J Gastroenterol 2013;108:1426-1438.

3. Androulakis I, Zavos C, Christopoulos P, Mastorakos G, Gazouli M. Safety of anti-tumor necrosis factor therapy during pregnancy in patients with inflammatory bowel disease. World J Gastroenterol 2015;21:13205-13211.

4. Guiddir T, Frémond ML, Triki TB, et al. Anti-TNF- $\alpha$ therapy may cause neonatal neutropenia. Pediatrics 2014;134:e1189-e1193.

5. de Meij TG, Jharap B, Kneepkens CM, et al. Long-term followup of children exposed intrauterine to maternal thiopurine therapy during pregnancy in females with inflammatory bowel disease. Aliment Pharmacol Ther 2013;38:38-43.

6. Akbari M, Shah S, Velayos FS, Mahadevan U, Cheifetz AS. Systematic review and meta-analysis on the effects of thiopurines on birth outcomes from female and male patients with inflammatory bowel disease. Inflamm Bowel Dis 2013;19:15-22.

7. Komoto S, Motoya S, Nishiwaki Y, et al. Pregnancy outcome in women with inflammatory bowel disease treated with antitumor necrosis factor and/or thiopurine therapy: a multicenter study from Japan. Intest Res 2016;14:139-145. 